\title{
The challenges of lower urinary tract symptoms in men
}

\author{
Greg Bailly, MD, FRCSC; ${ }^{*}$ J. Curtis Nickel, MD, FRCSC ${ }^{+}$
}

*Associate Professor and Residency Program Director, Department of Urology, Dalhousie University, Halifax, NS; †Professor, Department of Urology, Queen's University and Tier 1 Canadian Institutes of Health Research (CIHR) Canada Research Chair in Urologic Pain and Inflammation, Kingston, ON

Cite as: Can Urol Assoc J 2012;6(5):S129. http://dx.doi.org/10.5489/cuaj.12200

T wo distinct urologic entities were discussed in depth at the $2^{\text {nd }}$ Annual Canadian Urology Forum (2012): stress urinary incontinence (SUI) in women; and lower urinary tract symptoms (LUTS) in men.

The LUTS component of the educational program included a distinguished panel of Canadian and international experts, who collaborated to present and discuss the most up-to-date, evidencebased medicine and to share their own clinical experiences.

The overall learning objectives of this session were to:

- Recognize and describe what constitutes LUTS in older male patients;

- Define the roles of the prostate, bladder and other organs associated with LUTS;

- Identify the major knowledge gaps in the management of male patients who are experiencing LUTS; and

- Assess the evidence for currently available medical and interventional options to treat LUTS.

The faculty members presented six individual topics during the LUTS educational sessions. Dr. Claus Roehrborn, Professor and Chair in the Department of Urology at the University of Texas Southwestern Medical Center at Dallas, Texas, provided an overview of clinical practice guidelines for LUTS and benign prostatic hyperplasia (BPH). Dr. J. Curtis Nickel, Professor in the Department of Urology at Queen's University in Kingston, Ontario, and Tier 1 Canadian Institutes of Health Research Canada Research Chair in Urologic Pain and Inflammation, led a thought-provoking ses- sion on LUTS associated with prostatitis. Prof. Marcus Drake, Senior Lecturer in Urology at the University of Bristol, Bristol, United Kingdom, presided over two separate presentations. The first was an examination of male LUTS associated with overactive bladder, while the second was a discussion of "The Dilemma of Nocturia." Dr. Victor Nitti, Professor of Urology and Obstetrics and Gynecology and the Vice Chairman of the Department of Urology at New York University (NYU) Langone Medical Center, provided an overview of the appropriate workup of a man presenting with LUTS. Finally, Dr. Mostafa Elhilali presented a comprehensive overview of the various surgical treatment options for benign prostatic hyperplasia. Summaries of each of these presentations are provided in the following pages of this supplement.

At the conclusion of the above-mentioned lectures and associated discussion sessions, the faculty and participants again interacted through the discussion a complex case study detailing the appropriate management of a male patient with LUTS. The highlights of this discussion are also captured in the following pages.

The steering committee for the Canadian Urology Forum 2012 hopes that this document provides valuable information on LUTS in men to Canadian health-care professionals and their peers around the world.

Competing interests: Dr. Bailly is a paid consultant with Astellas, Allergan, Pfizer, and Eli Lilly.

Correspondence: Dr. Greg Bailly, 620-5991 Spring Garden Rd, Halifax, NS B3H 1Y4; gbaill@@dal.ca; Dr. J. Curtis Nickel, 76 Stuart Street in Kingston ON K7L 2V7; in@@queensu.ca 